\title{
The natural history of histologically proved drug induced liver disease
}

\author{
P G Aithal, C P Day
}

\begin{abstract}
Background-The long term outcome of drug related liver disease is unknown. Aims-To study the natural history of histologically proved drug induced hepatotoxicity.

Methods-110 patients with liver biopsies coded either as drug induced liver disease or hepatitis/cholestasis of unknown aetiology were identified from hospital records 1978-1996. Review of case notes and histology identified 44 patients with definite drug induced hepatotoxicity. Forty surviving patients were invited to attend a follow up clinic. History, examination, full liver screen, and isotope and ultrasound liver scans were repeated in all patients. Repeat liver biopsies were offered to patients with abnormal liver tests. Results-Presentation at index biopsy was jaundice in 24 patients, abnormal liver tests in 17, and hepatic failure in three. Antibiotics $(n=13)$ and non-steroidal antiinflammatory drugs $(n=11)$ were the most common drugs implicated. Initial histology showed acute hepatitis in six, chronic hepatitis in 20, and cholestasis in 18. At 1-19 years (median 5 years) follow up, $13 / 33(39 \%)$ patients had persistent significant abnormalities in their liver blood tests and/or scans. Three of the five repeat liver biopsies performed showed significant abnormalities. Factors predicting persistence or development of chronic liver disease were fibrosis and continued exposure to the drug.

Conclusions-Drugs should be considered in the differential diagnosis of abnormal liver function and/or histology, as failure to withdraw the offending drug is associated with a high risk of persistent liver damage.

(Gut 1999;44:731-735)
\end{abstract}

Keywords: drugs; chronic active hepatitis; toxic hepatitis; diclofenac

Centre for Liver

Research, The Medical School, Framlington

Place,

Newcastle-upon-Tyne

NE2 4HH, UK

P G Aithal

C P Day

Correspondence to: Dr Day.

Accepted for publication 17 December 1998
Hepatotoxicity is a potential complication of most prescribed drugs, presumably due to the central role of the liver in drug metabolism. ${ }^{12}$ In a few cases the adverse liver reactions are predictable and dose dependent ${ }^{3}$; however for most drugs liver injury is idiosyncratic. Even though many drugs associated with a significant risk of hepatotoxicity have been replaced by apparently safer drugs, hepatic drug reactions are being increasingly reported, ${ }^{45}$ with drugs still accounting for $5 \%$ of cases of jaundice admitted to hospitals. ${ }^{6}{ }^{7}$ Despite its relative frequency, surprisingly little information is available on the long term outcome of drug induced liver disease. ${ }^{8}$ Potential reasons for this include: a failure to consider drugs as the primary cause of abnormal liver function leading to "missed" diagnoses; the difficulty in establishing a definite diagnosis of drug induced liver disease, ${ }^{9}$ particularly excluding other diagnoses; and the widely held belief that, with a few notable exceptions, such as chronic hepatitis secondary to dantrolene ${ }^{10}$ or flucloxacillin induced cholestasis, ${ }^{11}$ drug induced hepatotoxicity is reversible following drug withdrawal and, accordingly, patients may not require long term follow up. We have sought to determine the natural history of patients with histologically confirmed drug induced liver disease presenting to a large teaching hospital over an 18 year period.

\section{Patients and methods}

From computerised histological records, a list of patients was obtained in whom a histological diagnosis of either drug induced liver disease or cholestasis/hepatitis of unknown aetiology was reported between 1978 and 1996. The case notes of all patients were traced and the following information extracted: personal details, full medical history including presentation prior to liver biopsy, detailed drug and alcohol history, physical findings, full blood count (haemoglobin, leucocytes, and platelets), standard liver function tests (albumin, bilirubin, alanine aminotransferase, alkaline phosphatase, and $\gamma$ glutamyl transferase), hepatitis $\mathrm{B}$ and $\mathrm{C}$ serology (where available), autoantibody screen, liver ultrasound and isotope scan, and endoscopic retrograde cholangiopancreatography (ERCP) where indicated. Permission for the study was obtained from the Newcastleupon-Tyne Joint Ethics Committee. Details of each patient's present general practitioner were obtained from the Office of Population Census and Surveys (OPCS), London. Death certificates were obtained as indicated.

The diagnosis of drug induced hepatotoxicity was based on the following International Consensus Criteria ${ }^{12}$ :

(1) The time from drug intake and withdrawal to the apparent onset of the reaction was "suggestive" (5-90 days from initial drug intake) or "compatible" (less than five or more than 90 days from initial drug intake and not more than 15 days of drug withdrawal for "hepatocellular" reaction

Abbreviations used in this paper: ERCP,

endoscopic retrograde cholangiopancreatography; OR, odds ratio; $\mathrm{CH}$, chronic hepatitis; $\mathrm{AH}$, acute hepatitis; LFT, liver function test. 
and not more than 30 days of drug withdrawal for cholestatic reaction) with drug hepatotoxicity.

(2) Course of the reaction after cessation of the drug was "very suggestive" (decrease in the liver enzymes by at least $50 \%$ of the excess over the upper limit of normal within eight days) or "suggestive" (decrease in the liver enzymes by at least $50 \%$ within 30 days for "hepatocellular" reaction and 180 days for "cholestatic" reaction) of drug reaction.

(3) Alternative cause of the reaction had been excluded by detailed investigations, including a liver biopsy in each case.

(4) There was a positive response to rechallenge (at least a doubling of liver enzymes) when such information was available.

Reaction was classified "drug related" if all of the first three criteria were met or if two of the first three criteria were met in the presence of a positive rechallenge response. In addition to these well established criteria, patients were also scored using a recently validated clinical scale for the diagnosis of drug induced hepatitis. ${ }^{13}$ A score of 11 or more out of a maximum of 20 was required for inclusion in this study as a lower score makes drug hepatotoxicity an unlikely diagnosis. ${ }^{13}$ Patients with a suspected drug overdose were excluded.
For those patients with a diagnosis of drug hepatotoxicity, permission was sought from the family doctor for each living patient, and each was then invited to attend for a follow up appointment during 1997. Patients presenting during 1996 were only invited to attend follow up when at least a year had passed since their index biopsy. In the clinic each patient was seen by one of us (PGA) and a full history and physical examination was obtained. All previous blood tests, and isotope and ultrasound scans were repeated. Repeat liver biopsies were offered to all patients with abnormal liver blood tests. Comparison between discrete variables was performed by Fisher's exact test using the Instat 2 programme (GraphPad Software, San Diego, California, USA). Odds ratios (OR) were calculated where appropriate.

\section{Results}

Of 110 patients initially identified, 44 were eventually classified as having drug related hepatotoxicity. Figure 1 details the exclusions. The alternative cause of liver disease in 42/110 patients was underlying malignancy in 11 , transplant related complications in 17, alcoholic liver disease in six, autoimmune hepatitis in five, systemic sepsis in two, and ischaemic hepatitis in one patient. None of the included patients had positive hepatitis B or C serology

Table 1 Clinical details of 44 patients with drug induced liver disease

\begin{tabular}{|c|c|c|c|c|c|c|}
\hline Case no & Sex & Age (y) & Drug & Presentation & Histology & $\begin{array}{l}\text { Follow up } \\
\text { (y) }\end{array}$ \\
\hline 1 & $\mathrm{~F}$ & 24 & Diclofenac & Liver failure & $\mathrm{AH}$ & 5.2 \\
\hline 2 & $M$ & 30 & Minocycline & Jaundice & $\mathrm{AH}$ & 1.2 \\
\hline 3 & $M$ & 60 & Rifampicin & Jaundice & $\mathrm{AH}$ & 5 \\
\hline 4 & $M$ & 63 & Halothane & Jaundice & $\mathrm{AH}$, fibrosis & 19.5 \\
\hline 5 & $\mathrm{~F}$ & 48 & Phenelzine & Liver failure & $\mathrm{AH}$ & 6 \\
\hline 6 & $\mathrm{~F}$ & 36 & Diclofenac & Raised enzymes & $\mathrm{CH}$ & 4 \\
\hline 7 & $\mathrm{~F}$ & 54 & Diclofenac & Raised enzymes & $\mathrm{CH}$ & 3.5 \\
\hline 8 & $\mathrm{~F}$ & 49 & Diclofenac & Raised enzymes & $\mathrm{CH}$ & 7.7 \\
\hline 9 & $\mathrm{~F}$ & 46 & Buprenorphine & Raised enzymes & $\mathrm{CH}$, steatosis & 1.7 \\
\hline 10 & $\mathrm{~F}$ & 72 & Cimetidine & Raised enzymes & $\mathrm{CH}$ & 2 \\
\hline 11 & $\mathrm{~F}$ & 53 & Omeprazole & Raised enzymes & $\mathrm{CH}$ & 2.6 \\
\hline 12 & M & 50 & Rifampicin & Liver failure & $\mathrm{CH}$, fibrosis & 5 \\
\hline 13 & M & 73 & Tetracycline & Raised enzymes & $\mathrm{CH}$, fibrosis & 5.2 \\
\hline 14 & $\mathrm{~F}$ & 73 & Nitrofurantoin & Jaundice & $\mathrm{CH}$, cirrhosis & 11.9 \\
\hline 15 & $\mathrm{~F}$ & 42 & Dantrolene & Jaundice & $\mathrm{CH}$ & 8.3 \\
\hline 16 & $M$ & 36 & Sulpiride & Raised enzymes & $\mathrm{CH}$ & 2.3 \\
\hline 17 & M & 61 & Nifedepine & Raised enzymes & $\mathrm{CH}$ & 1 \\
\hline 18 & $\mathrm{~F}$ & 57 & Methyldopa & Raised enzymes & $\mathrm{CH}$ & 7.9 \\
\hline 19 & M & 40 & Amiodarone & Raised enzymes & $\mathrm{CH}$ & 5.6 \\
\hline 20 & $\mathrm{~F}$ & 49 & Propylthiouracil & Raised enzymes & $\mathrm{CH}$ & 3.3 \\
\hline 21 & $\mathrm{~F}$ & 70 & Co-amoxiclav & Jaundice & Cholestasis, ductopenia & 1.8 \\
\hline 22 & $\mathrm{~F}$ & 18 & Co-amoxiclav & Jaundice & Cholestasis & 5.7 \\
\hline 23 & $\mathrm{~F}$ & 33 & Co-amoxiclav & Jaundice & Cholestasis & 1 \\
\hline 24 & $\mathrm{~F}$ & 63 & Co-amoxiclav & Jaundice & Cholestasis & 3.4 \\
\hline 25 & $\mathrm{~F}$ & 43 & Nitrofurantoin & Jaundice & Cholestasis & 6.5 \\
\hline 26 & $\mathrm{~F}$ & 62 & Dextropropoxyphene & Jaundice & Cholestasis & 1 \\
\hline 27 & $\mathrm{~F}$ & 53 & Dextropropoxyphene & Jaundice & Cholestasis & 12.4 \\
\hline 28 & $M$ & 51 & Dextropropoxyphene & Jaundice & Cholestasis & 8.2 \\
\hline 29 & $\mathrm{~F}$ & 34 & Trinordil & Jaundice & Cholestasis & 12.8 \\
\hline 30 & $\mathrm{~F}$ & 33 & Co-trimoxazole & Jaundice & Cholestasis & 5.9 \\
\hline 31 & $M$ & 49 & Phenytoin & Raised enzymes & Cholestasis, fibrosis & 3.9 \\
\hline 32 & $\mathrm{~F}$ & 32 & Gold & Jaundice & Cholestasis & 2.8 \\
\hline 33 & M & 53 & Chlorpromazine & Cholestasis & Cholestasis, fibrosis & 4.5 \\
\hline 34 & M & 58 & Piroxicam & Raised enzymes & $\mathrm{CH}$, fibrosis & $2.5^{\star}$ \\
\hline 35 & $\mathrm{~F}$ & 68 & Co-amoxiclav & Jaundice & Cholestasis & $0.2^{\star}$ \\
\hline 36 & M & 44 & Procainamide & Jaundice & $\mathrm{CH}$, fibrosis & $8.5^{\star}$ \\
\hline 37 & $\mathrm{~F}$ & 43 & Carbamazepine & Jaundice & $\mathrm{CH}$, fibrosis & $9.5^{\star}$ \\
\hline 38 & $M$ & 62 & Perhexiline & Raised enzymes & $\mathrm{CH}$ & $3.8+$ \\
\hline 39 & $\mathrm{~F}$ & 75 & Thioridazine & Jaundice & $\mathrm{AH}$ & $0.2 \dagger$ \\
\hline 40 & M & 38 & Chlorpromazine & Raised enzymes & $\mathrm{CH}$ & $0.5 \dagger$ \\
\hline 41 & $M$ & 54 & Chlorpromazine & Jaundice & Cholestasis & $0.7 \dagger$ \\
\hline 42 & M & 39 & Amitriptyline & Jaundice & Cholestasis & $0.1 \dagger$ \\
\hline 43 & $M$ & 34 & Co-amoxiclav & Jaundice & Cholestasis & $0.2 \dagger$ \\
\hline 44 & M & 29 & Dextropropoxyphene & Jaundice & Cholestasis & $2.3 \dagger$ \\
\hline
\end{tabular}

$\star$ Patient deceased; ffollow up by the patient's own doctor, but not reviewed as part of the follow up study. 
Patients with drug induced liver disease or hepatitis/ cholestasis of uncertain aetiology 1978-96

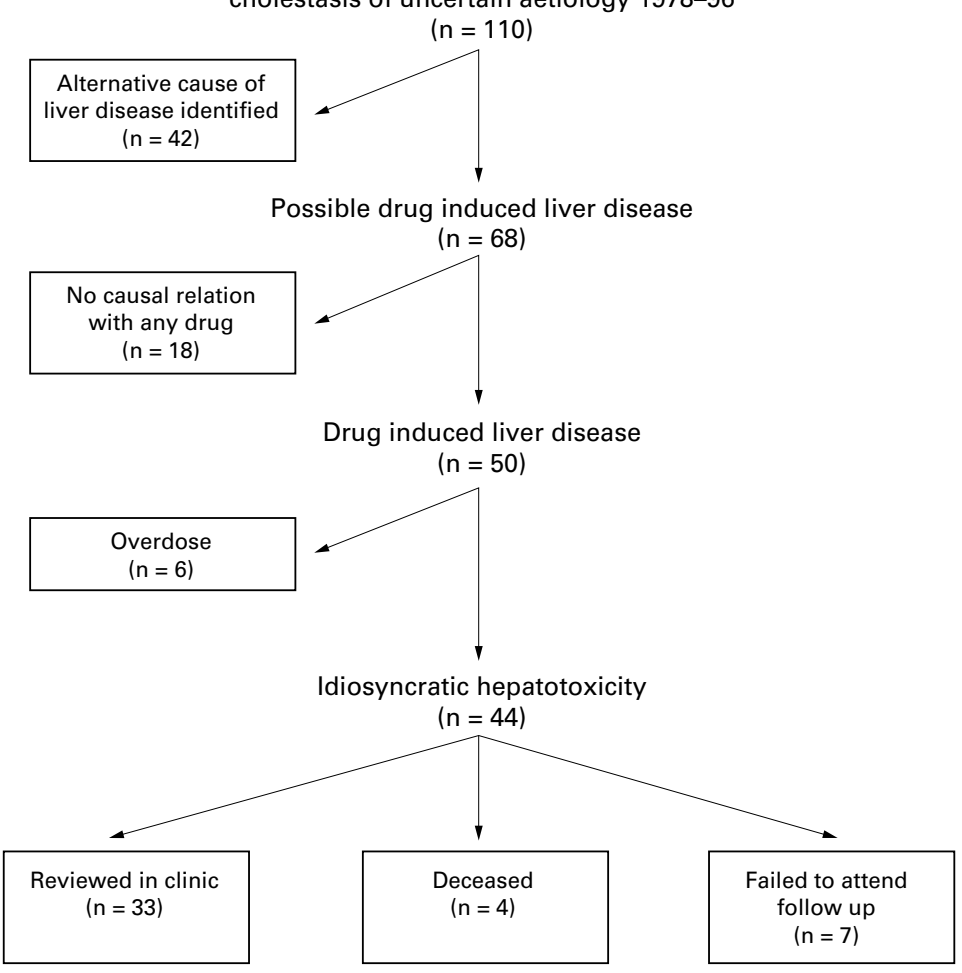

Figure 1 Patients with drug induced liver disease or hepatitis/cholestasis of uncertain aetiology, 1978-96; details of patients studied with reasons for exclusion from the study.

or autoantibodies associated with liver disease, although HCV serology was not performed in six patients who presented prior to the tests becoming available and who were either deceased $(n=3)$ or failed to attend follow up. The median age of the patients was 49 years (range 17-75) and two thirds were female (26/ 44). Table 1 lists the responsible drugs in each of the 44 patients. Antibiotics $(n=13)$ and analgesics/non-steroidal anti-inflammatory drugs $(n=11)$ accounted for over half the cases, with psychotropic drugs $(n=8)$ representing the other major group. The only specific drugs associated with more than two cases were co-amoxiclav (Augmentin) in six and chlorpromazine, diclofenac, and dextropropoxyphene in four cases each.

The initial presentation leading to the index liver biopsy was most commonly jaundice $(n=24)$, or abnormal liver blood tests performed as part of routine investigations $(n=17)$. A further three patients presented with hepatic failure manifest as hepatic encephalopathy. In only $17 / 44$ cases were drugs considered in the original differential diagnosis on the

Table 2 Initial laboratory tests in 44 patients according to histology

\begin{tabular}{lccc}
\hline & $C H(n=20)$ & $\begin{array}{l}\text { AH/cholestasis } \\
(n=24)\end{array}$ & $p$ Value \\
\hline Mean cell volume (fl) & $89.0(0.9)$ & $85.6(3.9)$ & 0.40 \\
Prothrombin time (s) & $14.0(0.5)$ & $13.2(0.6)$ & 0.32 \\
Total protein $(\mathrm{g} / \mathrm{l})$ & $72.1(1.6)$ & $67.4(1.8)$ & 0.06 \\
Albumin $(\mathrm{g} / \mathrm{l})$ & $41.4(1.4)$ & $39.8(1.1)$ & 0.19 \\
Bilirubin $(\mu \mathrm{mol} / \mathrm{l})$ & $51.6(22.7)$ & $179.5(34.3)$ & 0.002 \\
Alanine aminotransferase (U/1) & $114.5(31.2)$ & $287.5(80.0)$ & 0.05 \\
Alkaline phosphatase (U/l) & $252.7(31.1)$ & $355.6(43.1)$ & 0.03
\end{tabular}

Results expressed as mean (SE).

$\mathrm{CH}$, chronic hepatitis; $\mathrm{AH}$, acute hepatitis. histology request form. Drug induced liver diseases were classified entirely on the basis of the histological findings as recommended by the International Consensus Group. ${ }^{12}$ Initial histology showed features of acute hepatitis in six patients, chronic hepatitis in 20 , and predominant cholestasis in 18 with or without hepatitis. The histology result led the supervising clinician to the diagnosis of drug induced liver disease in a further 20 cases, leaving seven cases unrecognised for at least six months (five with chronic hepatitis and two with cholestasis and fibrosis). The vast majority of patients with acute hepatitis or cholestasis presented with jaundice and/or hepatic failure (22/24) compared with only a quarter of patients $(5 / 20)$ with chronic hepatitis $(\mathrm{p}<0.0001$, OR 33 (5.6193.1)). Consistent with this observation, both serum bilirubin and liver enzymes were significantly higher in patients with acute hepatitis/ cholestasis compared with those with chronic hepatitis (table 2).

At the time of follow up 40 patients were alive and 33 of these attended clinic. Of the remaining seven patients, three had moved away from the region and four were unwilling to take part in the study. At a median of five years follow up (range 1-19 years) no patient had presented with hepatic decompensation or had developed clinical signs of chronic liver disease. However, eight patients had persistent abnormalities in standard liver blood tests with or without associated scan (isotope/ ultrasound) abnormalities, and an additional five patients had persistently abnormal scans (isotope and/or ultrasound) without any elevation in liver enzymes (table 3). Five of the eight patients with abnormal liver tests underwent repeat liver biopsies, two patients refused, and one was taking long term anticoagulants for recurrent ventricular tachycardia. Three of the five biopsies showed significant residual pathology including chronic hepatitis, fibrosis, and ductopenia, although in no case had the histology increased in severity. The three patients with abnormal liver blood tests who were not biopsied all had abnormal isotope and/or ultrasound scans and seem likely to have underlying chronic liver disease. The five patients with abnormal scans (uneven uptake on isotope scans in three, shrunken liver or hepatomegaly with irregular texture on ultrasound scans in two) and normal liver blood tests seem likely to have inactive chronic liver disease. Liver disease did not appear on the death certificate of any of the four deceased patients, although clearly this does not definitely exclude the presence of chronic liver disease, particularly as three of these patients had chronic hepatitis and fibrosis on their index biopsies.

Features on presentation associated with persistence or development of chronic liver disease (determined histologically or assumed in patients with abnormal scans with or without abnormal liver blood tests) were: fibrosis at initial biopsy (6/7 versus $5 / 26$ without this lesion; OR 25.2 (2.4-259.4)); and continued exposure to the offending drug more than six months after the initial biopsy (5/7 versus $6 / 26$ with prompt withdrawal of drug; 
Table 3 Patients with abnormal liver blood tests or scans at follow up

\begin{tabular}{|c|c|c|c|c|c|c|}
\hline Case no & Drug & $\begin{array}{l}\text { Follow up after } \\
\text { drug withdrawal } \\
(y)\end{array}$ & LFTs & Scans* & Initial histology & Follow up histology \\
\hline 4 & Halothane & 19.5 & Normal & Abnormal & $\mathrm{AH}$ & - \\
\hline 7 & Diclofenac & 2.5 & Normal & Abnormal & $\mathrm{CH}$ & - \\
\hline 8 & Diclofenac & 5.7 & Abnormal & Normal & $\mathrm{CH}$ & Mild inflammation \\
\hline 9 & Buprenorphine & 1.7 & Abnormal & Abnormal & $\mathrm{CH}$, steatosis & Steatosis \\
\hline 12 & Rifampicin & 5 & Normal & Abnormal & $\mathrm{CH}$, fibrosis & - \\
\hline 13 & Tetracycline & 3.2 & Abnormal & Abnormal & $\mathrm{CH}$, fibrosis & $\mathrm{CH}$, fibrosis \\
\hline 14 & Nitrofurantoin & 11.9 & Normal & Abnormal & $\mathrm{CH}$, cirrhosis & - \\
\hline 16 & Sulpiride & 1.3 & Abnormal & Abnormal & $\mathrm{CH}$ & - \\
\hline 18 & Methyldopa & 7.9 & Abnormal & Abnormal & $\mathrm{CH}$ & $\mathrm{CH}$ \\
\hline 19 & Amiodorone & 3.6 & Abnormal & Abnormal & $\mathrm{CH}$, fibrosis & - \\
\hline 21 & Co-amoxiclav & 1.8 & Abnormal & Normal & Cholestasis, ductopenia & Ductopenia \\
\hline 24 & Co-amoxiclav & 3.4 & Normal & Abnormal & Cholestasis & - \\
\hline 31 & Phenytoin & 2.5 & Abnormal & Abnormal & Cholestasis, fibrosis & - \\
\hline
\end{tabular}

*Isotope and ultrasound (at least one of the following three features was regarded as abnormal: uneven uptake on isotope scan; shrunken liver on ultrasound scan; or hepatomegaly with irregular texture on ultrasound scan).

LFTs, liver function tests; $\mathrm{CH}$, chronic hepatitis; $\mathrm{AH}$, acute hepatitis.

OR $8.3(1.3-54.4))$. More patients with chronic hepatitis on initial histology had persisting evidence of chronic liver disease compared with those without (7/15 versus $4 / 18$ ) but this difference was not significant (OR $3.1(0.7-13.8)$ ).

\section{Discussion}

Until now information on the long term outcome of idiosyncratic drug induced liver disease has been derived largely from case reports that have described persistent biochemical evidence of liver injury (raised aminotransferases) as well as development of decompensated cirrhosis 6-76 months after initial injury. ${ }^{14-17}$ Despite these reports, drug induced liver injury is believed to be largely reversible, even when the histological pattern of injury is chronic active hepatitis. ${ }^{18} 19$ The results of this current study of patients with histologically documented idiosyncratic drug induced liver injury challenge these beliefs. A third of patients $(11 / 33)$ had either biochemical, radiological, or histological evidence of liver disease a median of five years following their index liver biopsy, including 9/15 patients with chronic hepatitis, two of whom had persistent necroinflammation on biopsy. Importantly, patients in this study were not selected because of the severity of their initial liver injury, only on the basis of their having history and investigations including histology compatible with drug induced injury and excluding other diseases. It might be expected that the more severe cases presenting during the study period would not have been biopsied due to the presence of coagulopathy.

Other than the presence of pre-existing fibrosis, the factor most associated with an adverse outcome was continuation of the implicated drug for more than six months after the index biopsy: $5 / 7$ of these patients $(71 \%)$ had abnormal investigations on follow up compared with $6 / 26$ patients $(23 \%)$ who had had their drug stopped. This adverse effect of continuation or inadvertent resumption of drug intake has been reported previously, ${ }^{14} 1719$ and shows that prompt recognition of drugs as the cause of liver injury is the most important aspect of management in these patients. In this series, from a large teaching hospital, drugs were suspected as the cause of abnormal liver function warranting a liver biopsy in only 17/44 cases $(39 \%)$. Even when histology became available, a failure to attribute the injury to drugs led to their continuation in $7 / 33$ patients $(21 \%)$ for more than six months.

Three factors seem likely to account for this failure to recognise drugs as the cause of liver injury, a problem which is presumably not unique to our institution. The first of these is the changing pattern of drugs associated with hepatotoxicity. In the 1960 s chlorpromazine was the leading cause of drug induced jaundice, ${ }^{20}$ while in the last two decades halothane was the most frequently reported hepatotoxin. ${ }^{6}{ }^{7}$ As these and many other hepatotoxic drugs have largely been replaced by drugs with better side effect profiles, one might expect the incidence of hepatotoxicity to be decreasing. However, a huge increase in the absolute number of prescriptions of drugs with a relatively low risk of hepatotoxicity has lead to both an increase in the number and a change in the pattern of hepatic drug reactions reported. ${ }^{6721}$ This is well illustrated in this study where antibiotics and analgesics/nonsteroidal anti-inflammatory drugs were incriminated in more than $50 \%$ of patients. The risks of developing significant hepatic injury secondary to co-amoxiclav and diclofenac (which accounted for 10/44 patients in our study) have been estimated to be around 1 per 100000 persons exposed. ${ }^{22} 23$

The second factor accounting for a failure to recognise drugs as the cause of liver injury relates to the histological pattern of injury. In the 1970s drugs were numerically the most important cause of chronic hepatitis, accounting for $28-66 \%$ of cases. ${ }^{18}{ }^{24}$ However, the drugs responsible for the majority of these cases, such as methyldopa and nitrofurantoin, are used much less frequently, and it has recently been suggested that drugs now contribute to less than $1 \%$ of all cases of chronic hepatitis, ${ }^{18}$ with one study reporting only one case of drug (nitrofurantoin) induced chronic hepatitis from 1985 to $1995 .^{25}$ This type of information may contribute to a failure to consider drugs in the differential diagnosis of chronic hepatitis. Indeed in this present study five of the seven cases where the drug was continued for more than six months following liver biopsy were patients whose biopsy showed 
chronic hepatitis. We have identified 20 cases of drug induced chronic hepatitis due to a wide range of drugs (table 1). These cases accounted for $6.5 \%$ of all cases of histologically proven chronic hepatitis presenting to our institution over the same time period. ${ }^{26}$ The observation that 15 of these 20 patients were asymptomatic and were identified only after abnormal liver function tests were detected on routine blood testing suggests that many cases of drug induced chronic hepatitis go undiscovered and may eventually conceivably contribute to cases of "cryptogenic" cirrhosis. Clearly, drugs must continue to be considered in the differential diagnosis of this histological pattern of injury.

The third factor accounting for a failure to recognise drugs as the cause of liver injury is that it may be caused by relatively new drugs not widely known to be associated with hepatotoxicity. This reflects the inevitable time lag that exists between marketing a new drug and the recognition of a rare side effect which is observed only after several accumulated patient years. This is well illustrated by diclofenac which, after several years of marketing, is being increasingly associated with the development of chronic hepatitis ${ }^{2327-29}$ and accounts for three patients in this current study.

In conclusion, therapeutic drugs remain a significant cause of liver injury and can be associated with a variety of histological appearances including chronic hepatitis. The pattern of drugs causing liver damage is changing and is now most often due to a rare side effect of commonly prescribed drugs such as antibiotics and anti-inflammatory drugs, and to recently introduced drugs whose hepatotoxicity may not yet be established. A high index of suspicion should be maintained for any drug taken by a patient with evidence of liver injury, as failure to recognise and subsequently discontinue the offending agent is associated with a high risk of liver damage persisting even after the drug is eventually stopped.

This work was supported in part by the EUROHEPATOX Biomed 2 Programme of the European Union (contrac BMH4-CT96-0658).

1 Zimmerman HJ, Maddrey WC. Toxic and drug-induced hepatitis. In: Schiff L, Schiff ER, eds. Diseases of the liver. Philadelphia: Lippincott Company, 1993:707-83.

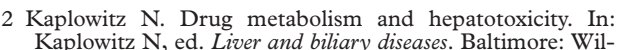
Kaplowitz N, ed. Liver and biliary
liams and Wilkins, 1992:82-97.

3 Mitchell JR, Jollow DJ, Potter WZ, et al. Acetaminopheninduced hepatic necrosis. IV. Protective role of glutathione. f Pharmacol Exp Ther 1973;187:211-17.

4 Miller RR. Hospital admissions due to adverse drug reactions: a report from Boston Collaborative Surveillance Program. Arch Intern Med 1974;134:219-23.

5 Lewis JH, Zimmerman HJ. Drug-induced liver disease. Med Clin North Am 1989;73:775-92.

6 Dossing M, Andreasen PB. Drug-induced liver disease in Denmark. An analysis of 572 cases of hepatotoxicity reported to Danish Board of Adverse Reactions to Drugs. Scand F Gastroenterol 1982;17:205-11.

7 Friis H, Andreasen PB. Drug-induced hepatic injury. An analysis of 1100 cases reported to the Danish committee on Adverse Drug Reactions between 1978 and 1987. F Intern Med 1992;232:133-8.

Med 1992;232:133-8. drug-induced liver injury. F Hepatol 1988;7:132-6.

9 Maddrey WC, Boitnott JK. Drug-induced chronic liver disease. Gastroenterology 1977;72:1348-53.

10 Utili R, Boitnott JK, Zimmerman HG. Dantrolene associated hepatic injury. Gastroenterology 1977;72:610-16.

11 Olsson R, Wiholm BE, Sand C, et al. Liver damage from flucloxacillin, cloxacillin and dicloxacillin. F Hepatol 1992; 15:154-61.

12 Benichou C. Criteria of drug-induced liver disorders. Report of an International Consensus Meeting. F Hepatol 1990;11:272-6.

13 Maria VAJ, Victorino RMM. Development and validation of a clinical scale for the diagnosis of drug-induced hepatitis. Hepatology 1997;26:664-9.

14 Maddrey WC, Boitnott JK. Isoniazid hepatitis. Ann Intern Med 1973;79:1-12.

15 Toghill PJ, Smith PG, Benton P, et al. Methyldopa liver Toghill PJ, Smith PG, Bento
damage. BMF 1974;3:545-8.

16 Ishak KG, Irey NS. Hepatic injury associated with the phenothiazines. Arch Pathol 1972;93:284-304.

17 Sharp JR, Ishak KG, Zimmerman HJ. Chronic active hepatitis and severe hepatic necrosis associated with nitrofurantoin. Ann Intern Med 1980;92:14-19.

18 Goldstein GB, Lam KC, Mistills SP. Drug-induced active chronic hepatitis. Am F Dig Dis 1973;18:177-84.

19 Seeff LB. Drug induced chronic liver disease, with emphasis on chronic active hepatitis. Semin Liver Dis 1981;1:104-15.

20 Cook GC, Sherlock S. Jaundice and its relation to therapeutic agents. Lancet 1965; i:175-9.

21 Almdal TP, Sorensen TIA. Incidence of parenchymal liver diseases in Denmark, 1981-1985: analysis of hospitalisation registry data. Hepatology 1991;13:650-5.

22 Farrell GC. Drug-induced cholestasis. In: Farrell GC, ed. Drug-induced liver disease. Edinburgh: Churchill Livingstone, 1994:319-70.

23 Banks AT, Zimmerman HJ, Ishak KG, et al. Diclofenacassociated hepatotoxicity: analysis of 180 cases reported to the Food and Drug Administration as adverse reactions. Hepatology 1995;22:820-7.

24 Lindberg J, Lindholm A, Lundin P, et al. Trigger factors and HLA analysis in chronic active hepatitis. BMF 1975;2:779.

25 Farrell GC. Drug-induced chronic active hepatitis. In: Farrell GC, ed. Drug-induced liver disease. Edinburgh: Churchill Livingstone, 1994:413-30.

26 Newton JL, Burt AD, Park JB, et al. Is the new international diagnostic scoring system for auto-immune disease too rigid [abstract]? Hepatology 1996;24:235A

27 Iverson TJ, Ryley NG, Kelly PMA, et al. Diclofenac associIverson TJ, Ryley NG, Kelly PMA, et al.
ated hepatitis. F Hepatol 1990;10:85-9.

28 Sallie RW, McKenzie T, Reed WD, et al. Diclofenac hepatitis. Aust NZ Z Med 1991;21:251-5.

29 Scully LJ, Clarke D, Barr RJ. Diclofenac-induced hepatitis in 3 cases with features of autoimmune chronic active hepatitis. Dig Dis Sci 1993;8:744-51. 